Revista

\title{
Multi-Ensayos
}

Vol. 7, núm. 14

ISSN: 2412-3285

https://multiensayos.unan.edu.ni

DOI: https://doi.org/10.5377/multiensayos.v7i14.12005

\section{La enseñanza-aprendizaje de la investigación bajo los nuevos paradigmas científicos}

\section{The teaching-learning of research under the new scientific paradigms}

\author{
Wilson Rojas Herrera ${ }^{1}$
}

Recibido: 03 de junio de 2021. Aceptado: 25 de junio de 2021

\section{RESUMEN}

Este ensayo presenta un análisis crítico sobre la forma en que se está enseñando la investigación a nivel de la educación superior universitaria. Se basa en cómo los nuevos paradigmas de la educación hacen válida la crítica, analizando las diferentes aristas que inciden, tanto en la universidad, como en los docentes y en los estudiantes, cuando se trata de la enseñanza-aprendizaje de la investigación. La idea es desmenuzar la realidad y conducir a inferencias que ayuden a desmitificar la investigación, como punto de partida, para un debate más amplio sobre las soluciones concretas.

Palabras clave: Investigación; aprendizaje; paradigmas; metodología; crítica.

\section{ABSTRACT}

This essay presents a critical analysis of the way research is being taught at the university higher education level. It is based on how the new paradigms of education make criticism valid, analyzing the different edges that affect, both in the university, as well as in the teachers and students, when it comes to the teaching-learning of research. The idea is to disentangle reality and lead to inferences that help demystify the research, as a starting point for a broader debate on concrete solutions.

Keywords: Research; learning; paradigms; methodology; criticism.

1 Profesor universitario, estudiante del doctorado en Educación en la Universidad La Salle, Costa Rica. Correo electrónico: wirojas@hotmail.com

(c) 2021 Revista Multi-Ensayos. 


\section{INTRODUCCIÓN}

A lo largo de mi experiencia como docente e investigador, he sido testigo de lo difícil que resulta enseñar a investigar a los estudiantes. Existe gran resistencia a elaborar investigaciones en los cursos y ni que decir cuando se trata de tesis o trabajos finales de graduación. La mayoría opta por caminos fáciles, como lo es el plagio, pagar por los trabajos o hacer "investigaciones" de último minuto para presentar algo, lo que transgrede, en cierta medida, la naturaleza misma de la investigación en la educación de los futuros profesionales del país. Con respecto a las causas, Belmonte (2002), señala que:

Frecuentemente, en el alumnado suele aparecer una visión peyorativa en la que se identifica investigar con una actividad semimisteriosa, necesitada de una gran cantidad de recursos y medios para poder ser realizada y apta únicamente para mentes privilegiadas y, por lo tanto, inaccesible para él. (p. 13).

La verdad es que existen una serie de mitos e ideas preconcebidas que se tejen alrededor de la labor investigativa en los estudiantes. Además de los aportados por Belmonte se podría mencionar que la metodología de la investigación es considerada aburrida, que no tiene algún valor o que requiere de un tedioso trabajo interminable que llega a fastidiar en demasía e interrumpe la labor de aprender y de gozar el proceso.

El propósito de este trabajo es poder analizar esta situación y tratar de desmitificar a la investigación, al presentar los problemas que la aquejan y algunas ideas de cómo debería ser aprender a investigar. La investigación no tiene por qué ser tediosa, aburrida o inalcanzable. Eso le devolvería su verdadero valor ante los ojos de los discentes y recobraría fuerzas para cumplir con la labor para la que fue creada.

\section{DESARROLLO}

Definitivamente, la clave del cambio está en poder entender el panorama actual de la enseñanzaaprendizaje de la investigación y tratar de liberar el poder creador y transformador que los estudiantes poseen.

A la luz de los nuevos paradigmas de esta nueva era, se torna interesante lo que apunta Spangler, mencionado por Huxley, et al. (1991), cuando se refiere a ello de la siguiente forma:

Considero que la Nueva Era, más que un acontecimiento futuro, es una metáfora para la expresión de un espíritu transformador y creativo... para situarnos en el mundo de una manera que nos abra a la presencia de lo divino -la presencia del amor -y de lo posible- en medio de nuestra mediocridad. (p. 23)

Una forma interesante de poder abrir la mente de los estudiantes es presentarles la disciplina de la investigación como algo "divino", es decir, el amor por lo que se hace. Gran parte del problema radica también en que no se ama la investigación. No hay nada más tedioso que investigar algo que no llama la atención, que no se vislumbra como un aporte concreto y verdadero, o que se hace por el mero requisito de hacerlo.

Además de ello, es importante poder criticar metodologías arcaicas y cuadradas que lo único que logran es abrumar al investigador y obligarlo a apegarse al "método", es decir, a un protocolo de investigación 
dado per se en el cual encajan cualquier tipo de investigación, sin importar el enfoque, tema o campo profesional, y sin dejar espacio para la creación y la transformación de su nuevo conocimiento. Así como lo afirma Fregtman, mencionado por Huxley, et al. (1991), al decir que:

El paradigma científico occidental describió al universo como un sistema mecánico-material de alta complejidad, reemplazando a la filosofía y a la teología como principios directrices de la existencia humana. En oposición a estas afirmaciones, la filosofía noética (del griego nous, espíritu) de antiguas culturas, considera la conciencia y la inteligencia creadora como atributos inmanentes y trascendentes al mundo fenoménico («explicado o explícito») como raíces primarias de la existencia. (p. 50)

La idea no es oponerse al "método", sino buscar uno que permita el libre flujo del conocimiento como un asombroso descubrimiento. Que exista una especie de unión, entre el método y el desarrollo investigativo. Ambas no deben estar supeditadas una con otra. Es lograr una conjunción hermosa entre aplicar el proceso investigativo con alegría y celeridad, y obtener resultados que permitan al individuo gozar del proceso y no solo de los resultados.

Ahora bien, ¿Cómo lograr sacar a la luz todo el potencial que tiene el estudiante en cuanto a investigación se trata? ¿Cómo lograr que venza sus temores y hastío ante un vasto universo que se abre ante sus ojos, pero se niega a ver? ¿Cómo poder enamorar al estudiante para que deje de lado su miopía y abrace la investigación? Tal y como lo afirmaba Reich, aunque él lo direccionaba al campo de la medicina, resulta ser tarea del educador también, el lograr tal cometido ya que "Esta es nuestra gran obligación: capacitar al animal humano para que acepte la naturaleza que existe dentro de él, para que deje de escapar de ella y goce de lo que ahora tanto teme." (p. 406)

Resulta fácil cuando se pone en los términos de Reich, sin embargo, no es tan sencillo cuando se lucha contra un sistema que preconiza el establecimiento de normas y reglas complicadas que deben ser seguidas al pie de la letra, y peor aún, cuando no se explica el fundamento de dichas reglas. Es difícil cuando se trata de seguir metodologías rígidas que impiden el libre pensamiento y que no dejan que exista una apertura más humana hacia la búsqueda del conocimiento; es más, se supedita ese conocimiento a la forma en que se obtuvo. Huxley, et al. (1991), lo expresa claramente al ilustrar la forma en que los estudiantes se sienten atrapados por el sistema:

Illich desarrolla una intuición que casi todos tuvimos en la escuela, que estábamos perdiendo el tiempo y adaptándonos a la burocracia del sistema. Considera que sirve más para mantener el statu quo que para fomentar el desarrollo de las personas, señala que las cosas importantes las aprende uno mismo (incluso se aprendería a leer espontáneamente, hacia los 9 años, viviendo como hoy rodeados de letras) y propugna una sociedad con el mínimo de escolarización posible (tampoco se trata de que los niños se queden en casa viendo la tele -la tele y nuestros hogares urbanos son también atacados por Illich). (p. 63)

¿Está la investigación supeditada a un sistema que trata de mantener el statu quo? ¿Qué tipo de statu quo trata el sistema de perpetuar? Esto podría ser materia para otro debate, sin embargo, es innegable que, todas las universidades siguen los mismos esquemas de investigación, se apegan a los mismos paradigmas ancestrales, enseñan las mismas metodologías y gradúan profesionales que cumplan con los requisitos ya preestablecidos. No es que se esté criticando la forma de enseñar y reglamentar la educación en las universidades, sino de cuestionar el por qué no hay un cambio significativo que traiga 
nuevas propuestas más acorde con los tiempos modernos, con las expectativas de los estudiantes y con el tipo de profesionales que demanda el contexto.

Ya lo expone Jiménez (2013) al mencionar lo que denomina statu quo universitario al definirlo como "Orden universitario establecido (statu quo universitario) y legitimado por los agentes-fuerzas internos y externos, como el modelo de universidad que así "debe ser" (el cual puede descomponerse en subórdenes: académico, económico, político, etc."

También lo exponía Espinoza (2005), mencionando a Feyerabend (1993), cuando contempla que otro de los males que aqueja a la universidad (y a la investigación como tal) actuales es haberse convertido en negocio, "La ciencia del siglo XX ha renunciado a toda pretensión filosófica y ha pasado a ser un gran negocio. Ya no constituye una amenaza para la sociedad, sino que es uno de sus puntales más firmes". (p. 83)

¿De dónde proviene dicha rigidez en los procesos? Precisamente, tiene su origen en el pensamiento Newtoniano que se basaba en la comprobación de los hechos, el apego a lo medible, aplicable a todo tipo de fenómenos, ya sean de índole físico o no, natural o artificial, focalizado o integral; como lo explica Capra (1992) al decir que: "La ciencia del siglo XVII se basaba en un nuevo método de investigación, defendido enérgicamente por Francis Bacon, que incluía dos teorías: la descripción matemática de la naturaleza y el método analítico de razonamiento concebido por el genio cartesiano" (p. 27), lo anterior lleva a cuestionarse ¿No hay campo para otro tipo de pensamiento?

Además de todo lo anterior, en muchos centros de estudio, se llega a tener al final una acumulación de "investigaciones" que se hacen por el mero requisito y que han desvirtuado el valor que la investigación reviste, kilómetros de estantes que albergan toneladas de tesis y trabajos finales de graduación los cuales, solamente vieron la luz cuando se expusieron para obtener un grado académico y que guardan polvo esperando ser desechados algún día. Es importante recobrar el sentido para poder lograr un cambio, una trascendencia, el poder ofrecer una solución válida a los problemas que el ser humano atraviesa y que, por medio del goce de investigar, se logre alcanzar metas certeras y válidas, las cuales sean publicadas, divulgadas, puestas a prueba, y que sirvan para continuar el ciclo del conocimiento. Es necesario, como lo apunta Maldonado (2013), concebir la investigación como: “...el hecho de que generalmente ha llegado a reconocerse que puede hablarse de progreso en la ciencia y en el conocimiento, pero no en términos acumulativos." (p. 16).

Tampoco se trata de generalizar, algunos centros de estudios han intentado cambiar sus esquemas con éxito, otros se resisten a ello y otros más lo han hecho sin llegar a un verdadero cambio. Por desgracia, la posibilidad de tener esa visión amplia raya en los intereses de muchos, se disfraza el proceso de importante, más en el fondo continúa ofreciendo pocas posibilidades de apertura, como lo señala Huxley, et al. (1991), al decir que:

Unas escuelas que pretenden ofrecer a los alumnos una formación personal íntegra, un atento despertar de la conciencia... aunque luego en la profesión que elijan acaben convertidos en matemáticos o campesinos. Un modelo de escuela ideal, pero, como es fácil de comprender, poco rentable para políticos y entornos sociales convencionales; un lujo demasiado caro para los tiempos que vivimos. (p. 75) 
Quizás el problema principal radica, no solo en estructura rígidas, sino en la incapacidad de poder tener esa visión más amplia que guíe los procesos de enseñanza-aprendizaje de la investigación, ya que, al tratarse de un universo complejo, se pierde dicha complejidad en cuestiones simplicista que nada aportan al fenómeno de la investigación. Es así como lo señala Huxley, et al. (1991), refiriéndose a Bateson, al decir que:

La mayoría de las incapacidades para aprender provienen de la ceguera perceptiva de pautas y estructuras, de la incapacidad para percibir las relaciones o el sentido de determinado grupo de elementos. Nuestras escuelas no nos ayudan -sugeriría Gregory- pues no nos enseñan a establecer conexiones y a percibir el contexto o entretejido ecológico de los sistemas en relación. (p. 67)

Si tan solo el estudiante dejara de preocuparse por el proceso y pudiera unir los puntos, es decir, ver la raíz, la motivación, el camino y el resultado en su conjunto, no como un fin en sí mismo, sino como el comienzo del cambio y el aporte hacia el futuro; quizás así el proceso investigativo perdería ese halo de misterio y se convertiría en algo más concreto, más definido, más vivencial para el estudiante.

Sería poder abrir los ojos a un nuevo paradigma, ver la investigación, no por lo que hace sino por lo que aporta, poder salir del encuadre y obtener diversas perspectivas que aporten más que el simple hecho de hacer una investigación, siguiendo un riguroso esquema que enclaustra y encadena el saber. Lo mencionaba Maldonado (2013) al expresar que: "pensar en términos de complejidad significa reconocer explícitamente que los fenómenos, sistemas o comportamientos complejos poseen más de una lógica y que pueden y deben ser explicados en términos perfectamente distintos a la lógica formal clásica". (p. 39).

O como lo expresa Capra (1992) al decir que:

La visión integral considera el mundo desde el punto de vista de las relaciones y las integraciones. Los sistemas están todos integrados y sus propiedades no pueden reducirse a las de unidades más pequeñas... En la naturaleza abundan los ejemplos de sistemas. Cada organismo... es un todo integrado, luego un sistema viviente... Otro aspecto importante de los sistemas es su naturaleza intrínsecamente dinámica. Las máquinas se construyen, mientras los organismos crecen. (p.143-144)

Es necesario lograr esa visión amplia e integral en el estudiante, que sienta que todo está interconectado, es mutuamente interdependiente y que su investigación sirve para unir los elementos dispersos, entendiéndolos en su justa dimensión. En pocas palabras, que no se trata de una investigación sin fundamento, esporádica, estática, higiénica y solitaria; y que su aporte traerá beneficio y relevancia, en la medida en que logre ampliar aún más esa visión integradora del saber.

Gran parte de la responsabilidad en la solución del problema, por no decir que quizás la única y más importante, radica en los docentes, quienes fuimos educados bajo un sistema newtoniano, lineal y mecanicista, y que ahora estamos trasmitiendo ese mismo sentido a los estudiantes. Son los educadores los que tienen el poder de dotar a los estudiantes de las posibilidades para que su intelecto emprenda el vuelo, sin apuros ni cortapisas, y que el cambio resurja como algo natural. Bien lo decía Morin, mencionado por Huxley, et al. (1991), al afirmar que: 
Es reconfortante alejarse para siempre de la palabra maestra que tiene una explicación para todo, de la lejanía que pretende resolverlo todo. Es reconfortante, en fin, considerar el mundo, la vida, el hombre, el conocimiento y la acción como sistemas abiertos. (p. 78)

¿Cómo lograr trasladar el proceso de enseñanza-aprendizaje de la investigación, de un monólogo donde el profesor habla y el estudiante escucha, a un sistema abierto donde todos los elementos revistan igual importancia y existan múltiples vías de comunicación y formas de hacer las cosas? ¿Cómo lograr dispersar esas ideas equivocadas de que el estudiante es un ser desprovisto de conocimiento alguno y que necesita del docente, en forma impositiva, para poder empezar a explotar el saber en él? Como lo menciona Capra (1992) al decir que:

Locke adoptó esta doctrina y, en una metáfora famosa, comparó la mente de un recién nacido a una tabula rasa, una pizarra en blanco sobre la cual se imprimiría el conocimiento una vez que fuese adquirido por medio de la experiencia sensible. (p. 35)

Los estudiantes no son tabulas rasas ni mucho menos, poseen una experiencia vicaria y personal, y un conocimiento albergado en ellos del cual se puede establecer el punto de partida. Precisamente, esa subestimación del estudiante es la que ha hecho que el sistema siga manteniendo el statu quo, y la enseñanza siga siendo tan rígida como hasta ahora. Definitivamente se necesita un cambio radical que abra el horizonte y las expectativas hacia nuevas inmensidades insospechadas y refresque la enseñanzaaprendizaje con nuevos bríos.

Para finalizar, es importante poder hacer énfasis en el investigador joven que el fin de sus investigaciones reviste un ideal más alto y profundo, que dichos esfuerzos investigativos adquieren relevancia cuando se trata de buscar el bien común y no el beneficio de unos pocos, y que la importancia de la investigación radica en el aporte de la misma, tal y como lo expresaba Fromm, al decir que: "Debería fomentarse el progreso científico y al mismo tiempo impedir que sus aplicaciones prácticas se convirtieran en un peligro para la especie humana. La investigación científica debe desvincularse de la industria..." (p. 100)

En otras palabras, no permitir que los frutos de la investigación profunda, concienzuda e integral sigan el rumbo para lo que muchas se han hecho hasta el día de hoy, servir a unos pocos. Buscar el bien común, y no convertir la investigación en algo como lo ejemplifica Capra (1992) al decir que: "Con Bacon la ciencia comenzó a tener como fin un tipo de conocimiento que permitiera dominar y controlar la naturaleza, conocimientos que hoy se emplean junto con la tecnología para lograr objetivos que son profundamente antiecológicos." (p. 28).

\section{CONCLUSIÓN}

Definitivamente hay mucho terreno que abarcar y mucho debate por hacer cuando se habla de la enseñanza-aprendizaje de la investigación en la educación superior.

Existen problemas de rigidez en los modelos que aprisionan al estudiante (y en cierta medida al docente) a apegarse a formatos establecidos que interrumpen el libre flujo de la creatividad y la expresión del espíritu en la investigación. Lo anterior, en aras de la "fiabilidad y seguridad" se sacrifican posibilidades 
de hacer diferentes las cosas. Existe también cierta reticencia por explorar otras vías, debido al temor o al confort que los "caminos recorridos" brindan.

Se ha convertido a la investigación, a través de un proceso tortuoso, en un producto que duerme el sueño de los justos en anaqueles polvorientos, logrando generar sinrazón y mal sabor de boca en los estudiantes, al obligarlos a adentrarse en los caminos de una investigación, con muchos esfuerzos y pocos resultados.

Se pretende desfragmentar la investigación (eso lo determinan los protocolos establecidos) sin tomar en cuenta el contexto, las fuerzas, los agentes involucrados y, sobre todo, el factor humano, generando una especie de tratado que científico que sirve para los propósitos académicos y nada más.

Es importante recalcar, al final, que no se pretende generalizar ni desechar por completo los intentos que muchos pensadores han introducido, al denunciar los hechos, ni tampoco el esfuerzo que muchas instituciones de educación superior realizan para mejorar sus procesos, sin embargo, quizás todavía falta mucho camino por recorrer.

\section{REFERENCIAS}

Belmonte, N. (2002). Enseñar a investigar. Orientaciones Prácticas. España: Ediciones Mensajero. ttps:// dialnet.unirioja.es/servlet/libro?codigo $=41355$

Capra, F. (1992). El punto crucial. Ciencia, sociedad y cultura naciente. [Versión PDF] Argentina: Editorial Estaciones. https://www.academia.edu/8141520/Capra_Fritjof_El_Punto_Crucial

Espinoza, J. (2005) La crítica de la ciencia moderna en la obra de P. Feyerabend y M. Berman. Revista. Filosofía Universidad de Costa Rica, XLIII Número doble (109/110), 81-85. http://www.inif.ucr. ac.cr/recursos/docs/Revista\%20de\%20Filosof\%C3\%ADa\%20UCR/Vol\%20XLIII/Nom.109-110/ La\%20critica\%20de\%20la\%20ciencia\%20moderna\%20en\%20la\%20obra\%20de\%20P.\%20 Fedeyerabend\%20y\%20M.\%20Berman.pdf

Fromm, E. (2013). Tener o ser. España: Fondo de Cultura Económica. https://docplayer.es/76681-Erichfromm-tener-y-ser.html

Huxley, A., Jung, C., Watts, A., Maslow, A., Fromm, E., Castaneda, C., Aurobindo, S., Graves, R., Schumacher, E., Krishnamurti, J., Kübler-Ross, E., Roszak, T., Rogers, C., Henderson, H., Capra, F., Bohm, D., WIlber, K., Grof, S. y Lovelock, J. (1991). Nueva Conciencia Plenitud personal y equilibrio planetario para el siglo XXI. Barcelona, España: Editorial Integral

Jiménez, Y. (2013). La construcción social del statu quo en la Universidad Nacional de México (19101914). Cultura y representaciones sociales, 8(15), 43-72. http://www.scielo.org.mx/scielo. php?script=sci_arttext\&pid=S2007-81102013000200002\&lng=es\&tlng=es.

Maldonado, C. (2013). Significado e impacto de las ciencias de la complejidad. [Versión PDF]. Colombia: Ediciones desde abajo. https://www.researchgate.net/publication/263652414_Significado_e_ Impacto_Social_de_las_Ciencias_de_la_Complejidad

Reich, W. (1957). Análisis del carácter. Argentina: Editorial Paidós. http://mastor.cl/blog/wp-content/ uploads/2014/09/Wilhelm-Reich-Analisis-Del-Caracter.pdf 